\title{
Auctioning the Right to Choose When Competition Persists
}

\author{
Ronald M. Harstad \\ Economics Department, University of Missouri, Columbia, Missouri 65211, \\ HarstadR@missouri.edu
}

First Draft: February, 2009

This Draft: July 8, 2009

\begin{abstract}
Several papers compare auctioning heterogeneous assets sequentially with sequentially selling the right to choose among assets not yet taken. Typically motivated by auctions of condos for owner occupation, these papers have assumed that each winning bidder exits, so each successive auction has less competition. In many heterogeneous-asset-sale situations, a winning bidder may still be interested in acquiring further assets. We build a simple model of persistent competition, in which the distribution of equilibrium revenue from separate sales is shown to be a mean-preserving spread of the distribution of revenue from selling rights to choose. Persistent competition reveals that a high bidder does not always select his most preferred asset, and that one asset being slightly more likely to be a favored asset discontinuously affects equilibrium bidding.

Key words: auction theory; rights-to-choose auctions; revenue comparisons; persistent competition; private information
\end{abstract}

\section{Introduction}

When a seller seeks to auction similar but distinct assets, it is commonplace in some settings and predominant in others to sell "rights to choose." In such an auction, each round determines the price at which the high bidder gets to select from those assets not yet taken. Ashenfelter and Genesove's (1992) exciting empirical paper on condominium conversion auctions has led to the handful of theoretical models that contrast rights-to-choose auctions with sequential auctions focusing primarily on this example. While illustrative, it is in an important aspect a limiting example. Gale and Hausch (1994), Burguet (2005), (2007), follow the condo conversion auctions that Ashenfelter and Genesove studied in assuming that a winning bidder ceases competing in later rounds, so that the level of competition is steadily decreasing. 
Condo auctions in Miami in recent years have not restricted bidding to those who intended to occupy a condo won in the auction, and have seen a bidder win, select a condo they intend to rent out, and continue competing. On many occasions, especially when several pieces from the estate of an art collector are being sold, art auctioneers have offered rights to choose, and a winning bidder choosing an artwork has remained to bid for further rights to choose. Some halfdozen recent mergers or acquisitions in the US banking industry have received regulatory approval only subject to a requirement that the newly combined entity divest a certain number of retail banking locations in key metropolitan areas where its dominance would otherwise be unacceptable. Our understanding of the resulting transactions is that they carry negotiation aspects making them less formal than the usual auction, but in some ways closely resemble right-tochoose auctions. As the number of branches sold typically is well in excess of the number of purchasing banks, clearly acquirers of some prime locations persist to compete for others. ${ }^{1}$

To illuminate persistent competition in right-to-choose auctions, we model the polar case: winning a right-to-choose not only leaves all bidders in competition, but all bidders, including the one now choosing an asset, have undiminished values (relative to the initial auction round) for any assets still in competition.

A key advantage of this modeling assumption is that a winning bidder's asset choice is open to strategic considerations. A winner who chooses and then exits obviously selects his asset. A winner who remains to compete for assets offered later decides whether to select his most-favored asset or to remove from competition an asset less highly valued by him in order to reduce the competitiveness in follow-up auctions.

Introducing this strategic dimension also serves to isolate a previously unexplored assumption, now found here to be a razor's edge case rather than robust. Prior papers have assumed that the identity of the most-highly-valued asset for a given bidder is determined equiprobably, making the assets homogenous from an ex-ante perspective. ${ }^{2}$ Initially, our model maintains this assumption. Under ex-ante homogeneity, the symmetric equilibrium bid function is the average of a bidder's values for the assets yet unchosen. In equilibrium, the favored asset is always chosen; expected revenue if the assets were sold separately is a mean-preserving spread of the expected revenue of selling rights to choose.

Both of these results are seen to be idiosyncractic consequences of the exante homogeneity assumption. Section 4 expands the model by assuming that each bidder draws the identity of his most-highly-valued asset from a common distribution that favors a particular asset, below called the "usual" favorite. The

\footnotetext{
${ }^{1}$ Our limited knowledge of all three examples stems primarily from private correspondence with participants in these markets; we have no data on the relative frequency of the rightto-choose format. The bank-branch sales involve a lengthy sequence of phone calls that correspond fairly closely to a series of Alternating Recognition auctions (Harstad and Rothkopf 2000).

${ }^{2}$ Gale and Hausch (1994), Burguet (2005, 2007) and Goeree, Plott and Wooders (2004) (this last is primarily an experimental paper) employ this assumption without comment on its robustness. Though types are drawn without replacement in the experimental study of Eliaz, Offerman and Schotter (2008), their assets are ex-ante homogeneous.
} 
outcome is strikingly different, and does not converge to the ex-ante-homogenous outcome as the preference distribution converges to uniform. The equilibrium bid function is a bidder's value of the usual favorite, whether or not the bidder in question values that asset more highly; equilibrium revenue now matches in distribution that from selling the assets separately. Most striking: the right to choose is always used to select the asset which would yield the greatest competition in the follow-up auction were it still available, even if the bidder selecting this asset values another asset far more highly, and even if the odds differential that the chosen asset would yield the greatest competition is arbitrarily small.

To sort out the role of ex-ante homogeneity, section 5 briefly discusses a model in which bidders typically diverge in their tastes. It also finds that the right to choose is always used to select the asset most likely to draw a higher rival bid in upcoming rounds. Section 6 discusses extensions, including moving beyond private values.

\section{A Model with Ex-Ante Homogeneity}

To focus on the issues just identified, this paper analyzes the two-asset, twobidder case. The two assets are labeled $\mathfrak{U}$ and $\mathfrak{R}$, to make the presentation in section 4 more mnemonic; for now, it may help to think of $\mathfrak{U}$ as a painting of an urban scene, $\mathfrak{R}$ as a painting of a rural scene. In keeping with the prior literature on rights-to-choose auctions, bidders' values will be private and independent (here, this will be seen below not to be a limiting assumption). Without loss of generality, the bidders will be numbered, and assets labeled, so that an analyst knows that bidder 1 has a higher value for asset $\mathfrak{U}, U_{1}$, than for asset $\mathfrak{R}, R_{1}$, although this convention is not knowledge of bidder 2. Bidder 2's values, $U_{2}$ and $R_{2}$, allow an analyst to distinguish two cases: $C$, Convergence, when the bidders concur as to which asset is more valuable (thus, $U_{2}>R_{2}$ ), and $D$, Divergence, when their tastes diverge: $U_{2}<R_{2}$. Of course, neither bidder knows whether they are in case $C$ or case $D$.

As in the prior literature, assume that a bidder's private knowledge is completely characterized by one scalar variable and one binary variable, evading the major complications of multi-dimensional signaling. Bidder $i=1,2$ privately observes random signal $X_{i} \sim G_{i}$ on $[0,1]$, and variable $F_{i} \in\{\mathfrak{U}, \mathfrak{R}\}$, equiprobably until section 4 , to maintain the ex-ante homogeneity assumption of prior papers. The interpretation is that $F_{i}$ is $i$ 's "favored" asset, the one with the higher value to him. Then if $F_{i}=\mathfrak{U}$ [correspondingly, $F_{i}=\mathfrak{R}$ ], $U_{i}=X_{i}$ $\left[R_{i}=X_{i}\right]$. Valuation specification is completed via a commonly known "lower value" function $L:[0,1] \rightarrow[0,1]$, with $L(0)=0,0<L^{\prime}(k)<1 \forall k \in[0,1]$. Thus, if $F_{i}=\mathfrak{U}$, $i$ 's asset values are $U_{i}=X_{i}, R_{i}=L\left(X_{i}\right)$; if $F_{i}=\mathfrak{R}$, $i$ 's asset values are $U_{i}=L\left(X_{i}\right), R_{i}=X_{i}$. The impact of this assumption is to make a higher-value bidder place a higher premium on obtaining his favored asset than does a lower-value bidder: $X_{1}>X_{2} \Leftrightarrow\left|U_{1}-R_{1}\right|>\left|U_{2}-R_{2}\right| .^{3}$ Recall that

\footnotetext{
${ }^{3}$ This assumption is introduced in Burguet (2005), and is more general than the assumptions regarding the ratio of (preferred value/less preferred value) in Gale and Hausch (1994),
} 
bidder $i$ obtaining $\mathfrak{U}[\mathfrak{R}]$ at price $p$ earns a profit of $U_{i}-p\left[R_{i}-p\right]$, regardless of whether the other asset is obtained.

The baseline auction simply sells the assets in separate auctions, English or second-price, in either order. Bidders have a dominant strategy to bid their value in each auction, whether risk-neutral or risk-averse, and expected revenue is $Y_{B}=E\left[\min \left\{U_{1}, U_{2}\right\}+\min \left\{R_{1}, R_{2}\right\}\right]$.

In the rights-to-choose auction, the high bidder in the first round pays the second-highest bid, and chooses one of the assets. The remaining asset is then auctioned off in round two by English or second-price auction, soon enough that negligible discounting occurs. Again, in round two, bidders have a dominant strategy to bid their value.

The symmetric equilibrium characterized next is in increasing strategies. Accordingly, a bidder winning the right to choose obtains no inference on learning his rival's bid as to his rival's favored asset. Thus, the rival's expected bid for the remaining asset is unaffected by which asset is selected.

It is straightforward to show that in equilibrium he selects his favored asset; under the homogeneity assumption, there will, on average, be just as strong competition in the following round no matter which asset he chooses.

Proposition 1 For both bidders to bid $b^{*}(U, R)=(U+R) / 2$ and choose their favored asset is an equilibrium. ${ }^{4}$

Proof. Let bidder 2 bid $b^{*}$, and consider bidder 1 upon observing values $\left(U_{1}, R_{1}\right)$. Note that $b^{*}$ is trivially a best response if the auction is not competitive (any undominated bid loses if $X_{1}<L\left(X_{2}\right)$; if $L\left(X_{1}\right)>X_{2}$, bidder 1 obtains both assets at the same expected price by bidding $b^{*}$ and choosing either asset). Hence, presume the auction is competitive: $X_{2}>L\left(X_{1}\right)$ and $X_{1}>L\left(X_{2}\right)$, so that each bidder can outbid the other on his favored asset if it is not also the other's favored asset. Suppose that by bidding $b^{*}$, bidder 1 will either outbid bidder 2 or will be awarded the right to choose due to a random tie-breaking mechanism. Temporarily assume (verified below) that bidder 1 chooses asset $\mathfrak{U}$. He pays price $p=b^{*}\left(U_{2}, R_{2}\right)$, attaining profit $U_{1}-\left(U_{2}+R_{2}\right) / 2 \geq\left(U_{1}-R_{1}\right) / 2$ in round 1 , with equality iff the bids are tied, and the strict inequality resulting from bidder 1 having the higher average value if his bid won outright. In the remaining round, the two equally likely cases yield as follows. $C$ : both bidders bid the value of their less-favored asset, bidder 1's profit is $\max \left\{0, R_{1}-R_{2}\right\}$, positive if his round 1 bid was higher, 0 if he tied. $D$ : bidder 2 bids for his favored asset and wins, with bidder 1 attaining 0 profit. Under this supposition, then, bidder 1's profit on both auctions is

$U_{1}-\left(U_{2}+R_{2}\right) / 2+\max \left\{0, R_{1}-R_{2}\right\} / 2 \geq\left(U_{1}-R_{1}\right) / 2+\max \left\{0, R_{1}-R_{2}\right\} / 2$, with equality iff the bids are tied.

Goeree, Plott and Wooders (2004), and Eliaz, Offerman and Schotter (2008). In sections 4 and 5 , this may be a conservative assumption.

${ }^{4}$ It is straightforward to adapt the method of proof in Levin and Harstad (1986) to show that this is the unique symmetric equilibrium in increasing strategies; details are omitted. 
Next, suppose the contrary: that by bidding $b^{*}$, bidder 1 will either be outbid by bidder 2 or will lose the right to choose due to a random tie-breaking mechanism. Bidder 1 earns 0 profit in round 1, and bidder 2 chooses his favorite asset (again a temporary assumption), yielding two equally likely cases. $C$ : bidder 2 chooses asset $\mathfrak{U}$, both bidders bid the value of their less-favored asset, bidder 1 's profit is $\max \left\{0, R_{1}-R_{2}\right\}$. $D$ : bidder 2 chooses asset $\mathfrak{R}$, and then bids against bidder 1 for 1 's favored asset; bidder 1 wins at price $U_{2}$, attaining profit $\left[U_{1}-L\left(X_{2}\right)\right]$. Under the contrary supposition, then, bidder 1 's profit on both auctions is $\left[U_{1}-L\left(X_{2}\right)\right] / 2+\max \left\{0, R_{1}-R_{2}\right\} / 2$.

Subtract bidder 1's profit when he does not get the right to choose from his profit when he does. If the round 1 bids tied, this difference is $\left(U_{1}-R_{1}\right) / 2-$ $\left[U_{1}-L\left(X_{2}\right)\right] / 2=0$, as tied bids require $X_{1}=X_{2}$, so he is indifferent over the tie-breaking outcome. If he outbid bidder 2 in round 1 , this difference is

$$
U_{1}-\left(U_{2}+R_{2}\right) / 2-\left[U_{1}-L\left(X_{2}\right)\right] / 2>\left(U_{1}-R_{1}\right) / 2-\left[U_{1}-L\left(X_{2}\right)\right] / 2>0
$$

so bidder 1 prefers to win in exactly those circumstances where bidding $b^{*}$ will win. Finally, if bidder 2 outbid him in round 1, this difference is

$$
U_{1}-\left(U_{2}+R_{2}\right) / 2-\left[U_{1}-L\left(X_{2}\right)\right] / 2<\left(U_{1}-R_{1}\right) / 2-\left[U_{1}-L\left(X_{2}\right)\right] / 2<0
$$

(both inequalities are reversed because bidder 1 being outbid implies $X_{1}<X_{2}$ ), so bidder 1 prefers to lose in exactly those circumstances where bidding $b^{*}$ will lose. A corresponding argument yields $b^{*}$ as a best response for bidder 2 .

It remains to demonstrate that a rights-to-choose winner choosing the favored asset is optimal. If he does so, his expected payoff is

$$
X_{1}-\frac{X_{2}+L\left(X_{2}\right)}{2}+\frac{1}{2}\left[L\left(X_{1}\right)-L\left(X_{2}\right)\right]
$$

as he only wins the followup auction in case $C$. If he instead chooses his lessfavored asset, his expected payoff reflects winning for sure in the followup auction:

$$
L\left(X_{1}\right)-\frac{X_{2}+L\left(X_{2}\right)}{2}+\frac{1}{2}\left[X_{1}-L\left(X_{2}\right)\right]+\frac{1}{2}\left[X_{1}-X_{2}\right]
$$

nonetheless, this expected payoff is lower when the auction is competitive.

\section{Revenue Comparison}

Expected revenue summed over the two rounds of the right-to-choose auction is calculated as follows. Round 1 expected revenue is

$$
E Y_{1}=E\left[\min \left\{\left(X_{1}+L\left(X_{1}\right)\right) / 2,\left(X_{2}+L\left(X_{2}\right)\right) / 2\right\}\right] .
$$

In case $C$ round 2 expected revenue is

$$
E Y_{2}^{C}=E\left[\min \left\{L\left(X_{1}\right), L\left(X_{2}\right)\right\}\right] .
$$




\begin{tabular}{|c|c|c|c|c|}
\hline \multicolumn{2}{|c|}{ Case C } & & \multicolumn{2}{c|}{ Case D } \\
\hline $\mathrm{U}_{1}$ & & & $\mathrm{U}_{1}$ & \\
\hline & $\left\{\mathrm{U}_{2}\right\}$ & & & $\mathrm{R}_{2}$ \\
\hline $\mathrm{Avg}_{1}$ & & & & \\
\hline & $\boldsymbol{A v g}_{2}$ & & & $\mathrm{Avg}_{1}$ \\
\hline & & & & \\
\hline $\mathrm{R}_{1}$ & & & $\left\{\boldsymbol{R}_{\mathbf{1}}\right\}$ & \\
\hline & $\left\{\boldsymbol{R}_{\mathbf{2}}\right\}$ & & & $\left\{\mathrm{U}_{2}\right\}$ \\
\hline
\end{tabular}

Figure 1: Revenue Comparison

Let $w \in\{1,2\}$ denote the round 1 winner, and $\neg w$ the loser. Then in case $D$, the round 1 loser is bidding for his favored asset, and expected revenue is

$$
E Y_{2}^{D}=E\left[\min \left\{X_{\neg w}, L\left(X_{w}\right)\right\}\right] .
$$

Combining, right-to-choose expected revenue is

$$
\begin{aligned}
E Y= & E\left[\min \left\{\frac{X_{1}+L\left(X_{1}\right)}{2}, \frac{X_{2}+L\left(X_{2}\right)}{2}\right\}\right] \\
& +\frac{E\left[\min \left\{L\left(X_{1}\right), L\left(X_{2}\right)\right\}\right]}{2}+\frac{E\left[\min \left\{X_{\neg w}, L\left(X_{w}\right)\right\}\right]}{2} .
\end{aligned}
$$

It is straightforward to see that expected revenue from separate auctions is

$$
\begin{aligned}
E Y_{B}= & E\left[\min \left\{U_{1}, U_{2}\right\}+\min \left\{R_{1}, R_{2}\right\}\right] \\
= & E\left[\min \left\{X_{1}, X_{2}\right\}+\min \left\{L\left(X_{1}\right), L\left(X_{2}\right)\right\}\right] / 2 \\
& +E\left[\min \left\{X_{1}, L\left(X_{2}\right)\right\}+\min \left\{L\left(X_{1}\right), X_{2}\right\}\right] / 2 .
\end{aligned}
$$

Thus, $Y_{B}$ is a mean-preserving spread of $Y$. To avoid over-involvement in notation, consider without loss of generality the case where the bidder winning the right to choose is labeled bidder 1 , and his favored asset continues to be $\mathfrak{U}$. Then the expected revenue comparison follows the illustration in Figure 1.

Variables shown higher in the figure represent higher expected values (per the labeling). Right-to-choose revenue $Y$ is half the sum of the four values shown in bold italic text. Separate auction revenue $Y_{B}$ is half the sum of the four values shown in brackets. So the expected average value of the right-tochoose loser appears in the right-to-choose revenue (twice, once in each case), but is replaced in the separate revenue, in case $C$ by that bidder's higher value and in case $D$ by that bidder's lower value. Hence, a risk-neutral seller is indifferent between auctioning the assets separately or auctioning the right to choose, while a risk-averse seller prefers the latter. 


\section{Ex-Ante Differentiation: A Usual Favorite}

Now consider a modification: each bidder is type $\mathfrak{U}$ with probability $\pi>1 / 2$, and type $\mathfrak{R}$ with probability $1-\pi$. That is, asset $\mathfrak{U}$ is "usually" a bidder's favored asset, $\mathfrak{R}$ "rarely" a bidder's favored asset. To illustrate bidding in this more complex environment, assume the distribution $G$ of bidders' values is uniform on $[0,1]$.

Now it is essential to consider bidding behavior separately for each type, as cases $C$ and $D$ have different probabilities and potentially different analyses for bidder types $\mathfrak{U}$ and $\mathfrak{R}$.

Comparable to the analysis of the equiprobable situation, an equilibrium is sought in which a bidder of type $f=\mathfrak{U}, \mathfrak{R}$ employs a bid function of the form $b_{f}(X)=w_{f} X+\left(1-w_{f}\right) L(X), w_{f} \in[0,1]$, a type-specific weighted average.

We will find that no equilibrium exists in which both types use the same weights. Hence, it is possible that $p$ (the price-setting bid in the auction for the right to choose) is more likely to have been submitted by one type of rival than by the other. Before selecting an asset, the winner rationally updates the probability that his rival is type $\mathfrak{U}$, from the ex-ante $\pi$ to

$$
\widehat{\pi}(p)=\frac{\pi g\left[b_{U}^{-1}(p)\right]}{\pi g\left[b_{U}^{-1}(p)\right]+(1-\pi) g\left[b_{R}^{-1}(p)\right]} .
$$

This will enter the winner's asset selection decisions below.

It is straightforward that a type $f=\mathfrak{U}$ bidder winning the right to choose selects his favored asset, $\mathfrak{U}$ : competition would likely be stronger were he to select $\mathfrak{R},{ }^{5}$ and his current-round payoff would be lower.

Initially suppose that a type $f=\mathfrak{R}$ bidder also selects his favored asset, $\mathfrak{R}$, in an equilibrium. Carefully following the steps in Proposition 1 that lead up to inequalities (1) and (2), but separately for the two types, and keeping track of the probabilities $\pi, 1-\pi$ and $\widehat{\pi}(p)$, leads to best response functions $w_{U}\left(w_{R}\right)$ and $w_{R}\left(w_{U}\right)$ that do not intersect. Hence, this initial supposition is inconsistent with an equilibrium of this form.

Instead suppose, then, that a type $f=\mathfrak{R}$ bidder selects his less-favored asset, $\mathfrak{U}$, in an equilibrium $\left(b_{U}, b_{R}\right)$. The above cases of convergence and divergence now require type-specific calculations, for cases $\mathfrak{U} C$, when he is type $\mathfrak{U}$ and types converge, $\mathfrak{U} D$, when he is type $\mathfrak{U}$ and types diverge, and $\mathfrak{R} C$ and $\mathfrak{R} D$, correspondingly. Conditional on a bidder drawing the indicated type, $\mathfrak{U} C$ and $\mathfrak{R} D$ have probability $\widehat{\pi}(p), \mathfrak{U} D$ and $\mathfrak{R} C$ probability $[1-\widehat{\pi}(p)]$.

To find such an equilibrium, as above, assume that the auction is competitive (it is straightforward that the equilibrium behavior remains a best response if it is not). Initially consider bidder 1 when he is of type $\mathfrak{U}$, and $w_{U} \in(0,1)$. If he wins the right to choose, and selects $\mathfrak{U}$, his payoff is

$$
\begin{aligned}
& X_{1}-\left\{\pi\left[w_{U} X_{2}+\left(1-w_{U}\right) L\left(X_{2}\right)\right]+(1-\pi)\left[w_{R} X_{2}+\left(1-w_{R}\right) L\left(X_{2}\right)\right]\right\} \\
& +\widehat{\pi}(p) \max \left\{0, L\left(X_{1}\right)-L\left(X_{2}\right)\right\}
\end{aligned}
$$

${ }^{5}$ We will show below that $\widehat{\pi}(p) \geq \pi>1 / 2 \forall p \in(0,1)$. 
where the subtracted term is the price of the right to choose, and the last term the expected payoff in the second auction, where competitiveness implies he is necessarily outbid in case $\mathfrak{U} D$, which has probability $[1-\widehat{\pi}(p)]$. If he loses the right to choose, his payoff is

$$
\pi \max \left\{0, L\left(X_{1}\right)-L\left(X_{2}\right)\right\},
$$

as his rival selects asset $\mathfrak{U}$ no matter what his type. Since this is smaller (equal only in the zero-probability event of a tie for right-to-choose) for any $w_{U} \in$ $(0,1),{ }^{6}$ bidder 1 of type $\mathfrak{U}$ improves his payoff by increasing $w_{U}$. Hence, an equilibrium requires $w_{U}=1$; that is, a bidder favoring the same asset usually favored bids his entire value of that asset for the right to choose, even if the chances that his rival favors the same asset only slightly exceed $1 / 2$.

Now consider bidder 1 when he is of type $\mathfrak{R}$, and $w_{R} \in(0,1)$. If he wins the right to choose, and selects $\mathfrak{U}$ (as the sought equilibrium requires), his payoff is

$$
\begin{aligned}
& L\left(X_{1}\right)-\left\{\pi X_{2}+(1-\pi)\left[w_{R} X_{2}+\left(1-w_{R}\right) L\left(X_{2}\right)\right]\right\} \\
& +\widehat{\pi}(p)\left[X_{1}-L\left(X_{2}\right)\right]+[1-\widehat{\pi}(p)] \max \left\{0, L\left(X_{1}\right)-L\left(X_{2}\right)\right\},
\end{aligned}
$$

where the case $\mathfrak{R} D$ (probability $\widehat{\pi}(p)$ ) has him bidding in the second round for his favored asset against a rival whose favorite asset has already been taken. If he loses the right to choose, his payoff is

$$
\pi\left[X_{1}-L\left(X_{2}\right)\right]+(1-\pi) \max \left\{0, L\left(X_{1}\right)-L\left(X_{2}\right)\right\},
$$

as his rival selects asset $\mathfrak{U}$ no matter what his type.

Next consider type $\mathfrak{R}$ 's choice between bidding weights $w_{R}$ and $w_{R}^{\prime}=w_{R} / 2$. With some probability, both bids win or both lose. However, in the event that the bidding weight choice $w_{R}$ wins and $w_{R}^{\prime}$ loses, the price in \{\} above necessarily exceeds $L\left(X_{1}\right)$, so bidder 1 of type $\mathfrak{R}$ prefers to lose. ${ }^{7}$ Consequently, only $w_{R}=0$ can be an equilibrium. As it is straightforward to show that bidder 1 of type $\mathfrak{R}$ cannot gain by selecting asset $\mathfrak{R}$ when $\left(w_{U}, w_{R}\right)=(1,0)$, this is then the only weighted-average equilibrium. ${ }^{8}$

The type of bidder whose favored asset is favored more often, however slightly, bids the full value of his favored asset, while the other type of bidder bids no more than the value of his less-favored asset. As the more frequently favored asset is selected whether it is the winning bidder's favored asset or not, the distribution of revenue is necessarily unaffected by switching between separate auctions and selling rights to choose.

\footnotetext{
${ }^{6}$ This relies on $\widehat{\pi}(p) \geq \pi$.

${ }^{7}$ At this point, as $w_{U}=1, \widehat{\pi}(p)=\pi g(p) /\left\{\pi g(p)+(1-\pi) g\left[b_{R}^{-1}(p)\right]\right\}$. In the relevant event $p \leq L(1)$ (and for somewhat higher $p$ ), $g(p)=g\left[b_{R}^{-1}(p)\right]$, so $\widehat{\pi}(p)=\pi$.

${ }^{8}$ Thus, if $\pi \in(0,1 / 2)$, correspondingly, both types bid their value $R_{i}$ of the rare asset, and select it if the right to choose is won.
} 


\section{Ex-Ante Homogeneity without Equiprobabil- ity}

Briefly consider a model (more artificial?) in which an analyst cannot find an ex-ante favorite, but instead of equiprobability, the chances that bidders diverge in their preference over assets exceeds one half. This can be done by adjusting the previous section's model to assume that bidder 1 is type $\mathfrak{U}$ with probability $\pi>1 / 2$, while bidder 2 is type $\mathfrak{R}$ with probability $\pi$. Thus, the ex-ante expected highest value of either asset is the same, but the probability that the two bidders favor different assets is $\pi^{2}+(1-\pi)^{2}>1 / 2$.

This model can be shown to have no equilibrium in undominated strategies which exhibits all types selecting their favored asset if winning the right to choose. An equilibrium exists in which all types select the asset most likely to be favored by the other bidder ( 1 selects $\mathfrak{R}, 2$ selects $\mathfrak{U})$. Thus, in equilibrium, type $\mathfrak{U}[\mathfrak{R}]$ of bidder $1[2]$ selects asset $\mathfrak{R}[\mathfrak{U}]$, despite favoring the other asset. This equilibrium yields less straightforward characterization: type $\mathfrak{R}[\mathfrak{U}]$ of bidder 1 (2] bids $\pi X+(1-\pi) L(X)$ for the right to choose; type $\mathfrak{U}[\mathfrak{R}]$ of bidder 1 (2] has a nonlinear bid function for the right to choose that is bounded below by $(1-\pi) X+\pi L(X)$ and above by $[X+L(X)] / 2$, and depends both on the underlying distributions of signals and the specific form of the $L$ function. The details are cumbersome, unilluminating, and omitted.

Note that, unlike the usual-favorite model, here a bidder planning to choose his favorite asset $(1 \mathfrak{R}$ or $2 \mathfrak{U})$ does not bid the full value of that asset. There, such a bidder knew that his favorite asset would be selected no matter which bidder won. Here, the rival bidder will select the other asset, so the right to choose is less valuable. Aggressive bidding comes from types not planning on selecting their favorite asset, but who know that their rival will select it. This model also becomes the section 2 model as $\pi \rightarrow 1 / 2$; interestingly, bids and revenue converge to that model, but asset selection does not.

\section{Remarks on Extensions}

Only notational complexity is affected by considerable weakening of value and distributional assumptions in the initial model. A distribution $\Gamma$ of $\left(X_{1}, X_{2}\right)$ can be asymmetric and affiliated; a bidder can value his favored asset at

$$
V=\omega X_{1}+(1-\omega) X_{2}, \text { for } \omega \in[1 / 2,1] .
$$

Hence, the equilibrium bidding, asset choice and revenue can be extended (via virtually identical proofs) to the general affiliated-values auction model of Milgrom and Weber (1982), and thus to common-value and affiliated-private-values auctions as special cases.

The models of sections 4 and 5 face dramatically added complexity if bidders' value estimates are not i.i.d. Given independent information, the results can readily be extended to the value function $V$ in (4), and thus to affiliated-values 
environments. A constraint limiting $\left[\sup _{x \in[0,1]} g(x)-\inf _{x \in[0,1]} g(x)\right]$ relative to $(\pi-1 / 2)$ would extend the results to distributions differing somewhat from the uniform.

Natural correspondents to the bid functions above would extend all results to risk-averse bidders, maintaining asset selections and presumably reducing revenue. In the initial model, a risk-averse seller might prefer sequential sales.

Selling more than two assets would add cumbersome but manageable complications, and presumably little insight (the second round of bidding, for the right to choose any asset but that chosen first, may well reveal both bidders' favoritism ranking across assets). Going beyond two bidders is notably problematic, as the expected competition-between the second-highest bidder for the right to choose, and highest rival bid by a rival whose tastes diverge with the winner-complicate asset choice, and thereby initial bidding, considerably.

\section{Conclusion}

This analysis has extended right-to-choose auctions in two important directions with respect to the inherited literature. First, removing the single-unit-demand constraint on bidders lets competition in rights-to-choose auctions persist and obtains revenue equivalence across auction formats. Second, weakening prior papers' assumption of ex-ante asset homogeneity allows for the possibility that a high bidder optimally selects the asset which will reduce competitiveness in follow-up rounds over his highest-valued asset. This selection occurs in equilibrium with probability approaching $1 / 2$ (in section 4 ). Critically, the equilibrium bid function in the more realistic case of ex-ante asset heterogeneity does not converge to the equilibrium bid function for the ex-ante homogeneous case as the degree of heterogeneity vanishes.

The discontinuity found above has a natural interpretation. The equilibria in sections 4 and 2 indicate that choosing the asset which would have led to the highest expected competitive bid in the next round (were it still available) is lexicographically more important than choosing the highest-valued asset. Since all bidders agree as to which asset to remove to minimize follow-up competition, it is known in advance which asset will be chosen first, and hence each bidder bids his value of that asset. However, in the razor's-edge case when favored assets are equiprobable, this lexicographically more important consideration is unavailable, and with it, certainty over which asset will be chosen first is unavailable. So each bidder reverts to the lexicographically secondary criterion, which is to bid the average value of assets currently available. Thus, choosing the highest-valued asset is trivial if not remaining in competition, but of secondary importance if the chooser's competition will persist.

The model considered briefly in section 5 shows some robustness of the greater importance of removing the asset which is expected to yield the greatest competition; in that model the favored asset is selected less than half the time.

Many markets where rights-to-choose auctions are used probably fit poorly to either the prior models or the polar opposite model here: neither is no further 
competition by a winning bidder guaranteed, nor is persisting competition with each winning bidder still bidding as strongly as if he had not won. ${ }^{9}$ These intermediate cases deserve analysis; this paper strikingly points to the importance of not limiting that analysis to the ex-ante-homogeneous case.

\section{Acknowledgments}

Vlad Mares has been more helpful than is readily expressed in a simple acknowledgment; conversations with him and his provocative questions led to a focus which yielded sections 4 and 5 . Two diligent referees have stimulated improvements in the exposition.

I have been stimulated, cajoled and inspired by Mike Rothkopf on countless occasions, and have gained substantially from my interactions with him and our work together. My writing first drafts and Mike acutely editing proved a valuable division of labor. He has been a tireless supporter of and believer in the social value of serious attempts to model economic and strategic phenomena. Mike first pointed to rights-to-choose auctions as a topic worthy of study prior to the earliest literature on the subject.

\section{References}

[1] Ashenfelter, O., D. Genesove. 1992. Testing for price anomalies in real-estate auctions. American Economic Review $\mathbf{8 2}$ 501-05.

[2] Burguet, R. 2005. The condominium problem: Auctions for substitutes. Review of Economic Design 9 73-90.

[3] Burguet, R. 2007. Right to choose in oral auctions. Economics Letters 95 167-73.

[4] Eliaz, K., T. Offerman, A. Schotter. 2008. Creating competition out of thin air: An experimental study of right-to-choose auctions. Games and Economic Behavior 62 383-416.

[5] Gale, I., Donald Hausch. 1994. Bottom-fishing and declining prices in sequential auctions. Games and Economic Behavior 7 318-31.

[6] Goeree, J. K., C. R. Plott, J. Wooders. 2004. Bidders' choice auctions: Raising revenues through the right to choose. Journal of the European Economic Association 2 504-15.

[7] Harstad, R. M., M. H. Rothkopf. 2000. An alternating recognition model of English auctions. Management Science 46 1-18.

\footnotetext{
${ }^{9} \mathrm{~A}$ high bidder may often continue to compete, but follow-up bidding may be influenced by marginal valuations that are reduced by assets already won (winning multiple condos in the same Miami building may reduce portfolio diversification), or by budget constraints; neither has been examined here.
} 
[8] Milgrom, P. R., R. J. Weber. 1982. A theory of auctions and competitive bidding. Econometrica 50 1089-1122. 